\title{
Tecnologias no Ensino e Aprendizagem de Deficientes Auditivos: Uma Revisão Sistemática de Literatura
}

\author{
Isabella Sakis $^{1}$, Felipe F. Lorenci ${ }^{1}$, Giliane Bernardi ${ }^{1}$ \\ ${ }^{1}$ Universidade Federal de Santa Maria (UFSM) \\ Santa Maria - RS - Brazil \\ \{isakis,giliane\}@inf.ufsm.br lorencifelipe@gmail.com
}

\begin{abstract}
The difficult educational inclusion situation of the hearing impaired in Brazil has been attenuated by public policies that universalize access. However, other measures, such as the use of Information and Communication Technologies (ICTS) in the classroom, should be taken to ensure the permanence and participation of these students. Thus, this Systematic Literature Review sought to discover what technologies are being used and developed in Brazil for this aim. With this review, great contributions were identified, such as educational games, pedagogical architectures and virtual learning environments, among other applications, directed mostly to elementary school students.
\end{abstract}

Resumo. A dificultosa situação da inclusão educacional de deficientes auditivos no Brasil tem sido atenuada por políticas públicas que universalizam o acesso. No entanto, outras medidas, como o uso de Tecnologias de Informação e Comunicação (TIC) em sala de aula, devem ser tomadas para assegurar a permanência e a participação desses alunos. Assim, essa Revisão Sistemática de Literatura buscou descobrir quais tecnologias estão sendo utilizadas e desenvolvidas no Brasil com esse propósito. Como resultados, foram identificadas diversificadas contribuições, como jogos educacionais, arquiteturas pedagógicas e ambientes virtuais de aprendizagem, entre outras aplicações, direcionadas, na maioria, a alunos do ensino fundamental.

\section{Introdução}

O direito à educação para todos é garantido pela Declaração Universal dos Direitos Humanos [ONU 1948] e corroborado no nosso país pela Constituição Brasileira de 1988 [Brasil 1988]. Apesar disso, a inclusão educacional de pessoas com deficiência, que foi vigorada oficialmente somente em 1994, por meio da adoção da Declaração de Salamanca [ONU 1994] às diretrizes educacionais do Brasil, ainda enfrenta dificuldades.

Conforme consta em [Unesco 1998], para garantir a igualdade no processo de ensino e aprendizagem de pessoas com deficiência, o acesso à educação deve ser universalizado, pois suas necessidades básicas de aprendizagem requerem atenção especial. Nesse sentido, políticas de inclusão educacional que visam reduzir barreiras aos educandos com deficiência, também lançam desafios e dificuldades, principalmente no tocante do Ensino Superior. Dessa maneira, para atenuar esse cenário, em 2012 foi instituído o sistema de reserva de vagas a deficientes no Brasil, através da Lei ${ }^{\circ} 12.711$, 
VII Congresso Brasileiro de Informática na Educação (CBIE 2018)

Anais do XXIX Simpósio Brasileiro de Informática na Educação (SBIE 2018)

que dispõe sobre o ingresso nas universidades federais e nas instituições federais de ensino técnico de nível médio [Brasil 2012].

Todavia, ampliar o acesso não é o bastante, pois garantir a presença do aluno em sala de aula não implica diretamente na sua continuidade acadêmica. Prova disto são as diminutas taxas de permanência e conclusão desses alunos nos cursos de Ensino Superior. De acordo com o último Resumo Técnico do Censo da Educação Superior do Brasil [INEP 2018], em 2015 ocorreram 38.837 matrículas de pessoas declaradas com deficiência e somente 5.312 concluintes. Em consonância a isso, o Grupo de Trabalho da Política Nacional de Educação Especial do Brasil afirma que, na educação superior, a inclusão educacional se efetiva por meio de ações que promovam o acesso, a permanência e a participação dos alunos [Brasil 2008].

Considerando o foco deste trabalho, observa-se ainda que o referido documento afirma que para inclusão dos alunos surdos, deve ser ofertada a educação bilíngue, que desenvolve o ensino escolar na Língua Brasileira de Sinais (LIBRAS) e na Língua Portuguesa, como segunda língua na modalidade escrita. Dessa forma, o atendimento educacional especializado deve ser ofertado nas duas modalidades e os sistemas de ensino devem disponibilizar instrutor de ensino bilíngue e tradutor/intérprete de LIBRAS.

No que tange a atuação dos intérpretes, [da Silva et al. 2016] afirmam que eles são responsáveis por promover a interação discursiva entre os surdos e ouvintes nas relações acadêmicas e sociais, mas que esse processo é complexo, devido às atribuições e competências necessárias para o exercício da função, principalmente na Educação Superior, na qual deve-se levar em conta a densidade lexical de conteúdos relacionados à formação universitária. Dessa forma, não se deve atribuir inteiramente a esses profissionais a responsabilidade de inserção dos alunos no âmbito acadêmico. Sendo assim, percebe-se que para assegurar e manter a inclusão educacional, é imprescindível que as instituições de ensino ofereçam recursos e materiais pedagógicos que possam auxiliar o aluno com deficiência dentro e fora da sala de aula.

Nesse contexto, as Tecnologias de Informação e Comunicação (TIC) estão sendo utilizadas como agentes transformadores no processo de ensino e aprendizagem de alunos com alguma deficiência. No caso dos deficientes auditivos, a promoção da aprendizagem pode ser ofertada de duas maneiras: direta, por meio de softwares educacionais que disponibilizam recursos pedagógicos, didáticos ou lúdicos para aquisição de conhecimento; e/ou indireta, com o uso de softwares que promovem a comunicação, interação e consequente inclusão desses alunos no âmbito escolar.

Em vista disso, realizou-se um Revisão Sistemática de Literatura (RSL) para identificar quais tecnologias estão sendo utilizadas e desenvolvidas no Brasil com o propósito de promover a inclusão educacional de deficientes auditivos. Dessa maneira, este artigo descreve o planejamento, condução e resultados da RSL, relatando contribuições que podem ser elencadas a partir da utilização das tecnologias encontradas, com ênfase especial nas que são utilizadas no Ensino Superior e/ou no ensino de computação.

\section{Trabalhos Correlatos}

Ao realizar a busca por estudos primários, relatada na próxima seção, foram encontradas outras RSLs semelhantes a essa, e as que não apresentaram nenhum relato de 
VII Congresso Brasileiro de Informática na Educação (CBIE 2018)

Anais do XXIX Simpósio Brasileiro de Informática na Educação (SBIE 2018)

desenvolvimento ou utilização de tecnologia foram desconsideradas no levantamento de dados. Assim, os objetivos e principais resultados de alguns destes estudos são relatados nesta seção, por considerá-los como trabalhos correlatos ao apresentado.

[Ramires et al. 2013] realizaram um mapeamento sistemático para catalogar os recursos de Tecnologia Assistiva (TA) que auxiliam as pessoas com deficiência no processo de ensino/aprendizagem. A partir dos trabalhos analisados foram catalogadas 153 tecnologias, que são ferramentas específicas ou adaptações de já existentes, Ambientes Virtuais de Aprendizagem (AVA) e jogos. Essa revisão, diferentemente da nossa, não teve seu foco em uma deficiência específica e verificou que todas as deficiências foram contempladas nos estudos, com grande concentração na auditiva e visual. Além disso, não são relatadas contribuições das tecnologias catalogadas.

Por outro lado, a revisão realizada por [Rocha et al. 2014] teve se enfoque em AVAs. A partir da análise dos 29 estudos selecionados, verificou-se que o tipo de AVA mais utilizado é o LMS - Learning Management System, sendo o vídeo o principal recurso educacional. Constatou-se também que 58,62\% das soluções são para adultos e $31,03 \%$ para crianças. Apesar da relevância dos resultados apresentados, considera-se que o mapeamento de outros tipos de tecnologias, além de AVAs, iria enriquecer o levantamento das contribuições.

Duas revisões mais recentes relatam sobre tecnologias que auxiliam no ensino de áreas específicas, diferentemente das anteriores. Em [Santos et al. 2016] foram pesquisadas soluções que ensinam LIBRAS e, a partir dos 27 estudos identificados, foi possível verificar as abordagens pedagógicas adotadas nos softwares catalogados. Já em [de Souza 2016] o propósito foi investigar quais tecnologias são utilizadas para o ensino da Geometria Interativa para deficientes. Dos 20 estudos primários selecionados, $85 \%$ são direcionados a deficientes visuais e $15 \%$ a deficientes auditivos, cujas soluções contribuem com interações gráficas para auxiliar na compreensão do conteúdo.

Dessa maneira, constatou-se alguns diferenciais entre esses trabalhos analisados e a nossa revisão, visto que esta investigou todas tecnologias que buscam auxiliar no ensino e aprendizagem de deficientes auditivos aplicadas em diferentes níveis e modalidades de ensino. Além disso, os objetivos dessa RSL a distingue das demais, ao deferir determinada atenção aos estudos que visam promover a aprendizagem de computação e aos direcionados ao ensino superior, apesar da abrangência pretendida em relação aos contextos educacionais e aos tipos de tecnologias.

\section{Revisão Sistemática de Literatura}

Revisão Sistemática de Literatura é um método definido por [Kitchenahm 2004] que permite, a partir da determinação de questões de pesquisa, o levantamento de dados necessários para respondê-las através da leitura e seleção de artigos. Essa seleção é guiada por critérios de inclusão e exclusão definidos no início da pesquisa, e constituem, juntamente com as questões, o protocolo de pesquisa.

\subsection{Planejamento}

Seguindo esse protocolo foram definidas quatro questões de pesquisa, que possibilitaram nortear a revisão, sendo que a primeira é a dúvida central e as outras dependem dos seus resultados. 
VII Congresso Brasileiro de Informática na Educação (CBIE 2018)

Anais do XXIX Simpósio Brasileiro de Informática na Educação (SBIE 2018)

QP1: "Que tecnologias têm sido utilizadas e desenvolvidas para auxiliar na inclusão educacional de deficientes auditivos?"

QP2: "Quais os principais objetivos das tecnologias identificadas na QP1?”

QP3: "Que níveis de ensino são público-alvo das tecnologias identificadas na QP1?"”

QP4: "Que contribuições são destacadas na educação de deficientes auditivos mediada por tais tecnologias?"

Além disso, foram definidos critérios de inclusão e exclusão para orientar na seleção dos estudos primários:

- Critério de Inclusão

I1: Estudos que apresentam soluções tecnológicas direcionadas aos deficientes auditivos.

\section{- Critérios de Exclusão}

E1: Estudos que não apresentam soluções tecnológicas direcionadas aos deficientes auditivos.

E2: Estudos que não apresentam desenvolvimento/proposta/uso de tecnologias.

E3: Estudos não disponíveis para leitura digital.

E4: Estudos equivalentes - escritos pelos mesmos autores e com relatos sobre a(s) mesma(s) tecnologia(s) (Foram mantidos os mais recentes).

E5: Estudos que não foram desenvolvidos no Brasil.

Finalmente, após o estabelecimento do protocolo de pesquisa, algumas estratégias de busca foram adotadas para coletar os estudos desejados, incluindo a string de busca, o período de publicação e as fontes dos dados, especificados a seguir.

- String de Busca: "auditiv*'” OR "audição" OR "surd*” OR "libras" OR "língua de sinais".

- Período de Publicação: de 2007 a 2017.

- Fontes de Dados: as fontes de dados escolhidas para realizar as pesquisas abrangeram os principais eventos e periódicos de informática na educação do Brasil: Simpósio Brasileiro de Informática na Educação (SBIE), Workshops do Congresso Brasileiro de Informática na Educação (WCBIE), Workshop de Informática na Escola (WIE), Revista Brasileira de Informática na Educação (RBIE), Revista Novas Tecnologias na Educação (RENOTE) e o Simpósio Brasileiro Sobre Fatores Humanos em Sistemas Computacionais (IHC).

A partir desse planejamento foi possível dar prosseguimento a condução da RSL, descrita na seção seguinte.

\subsection{Condução}

Uma revisão sistemática tem início pela seleção dos estudos primários. Então os estudos primários, encontrados na busca nos acervos digitais escolhidos, passaram por três fases de seleção, com a aplicação dos critérios de inclusão e exclusão definidos anteriormente. 
VII Congresso Brasileiro de Informática na Educação (CBIE 2018)

Anais do XXIX Simpósio Brasileiro de Informática na Educação (SBIE 2018)

$1^{\text {a }}$ Seleção: Aplicação dos critérios de inclusão, a partir da leitura do título, palavraschave e resumo.

$2^{\text {a }}$ Seleção: Aplicação dos critérios de exclusão, a partir da leitura da introdução e conclusão.

$3^{\text {a }}$ Seleção: Aplicação dos critérios de exclusão, a partir da leitura do artigo inteiro.

Ao realizar a busca dos estudos e $1^{\text {a }}$ seleção, foram encontrados 109 artigos. Em seguida, após a $2^{\mathrm{a}}$ seleção, restaram 83 artigos. E, finalmente, após a $3^{\mathrm{a}}$ seleção, restaram 57 artigos. Estes resultados estão representados na Figura 1. Com relação a evolução histórica das contribuições encontradas, os anos que mais tiveram estudos publicados são os de 2014 (10 estudos) e 2017 (14 estudos). Além disso, em 2015 houve uma grande quantidade de estudos que não puderam ser selecionados por não estarem disponíveis para leitura digital. Dessa maneira, percebe-se crescente interesse ao longo dos últimos anos em desenvolver e utilizar tecnologias no ensino de deficientes auditivos.

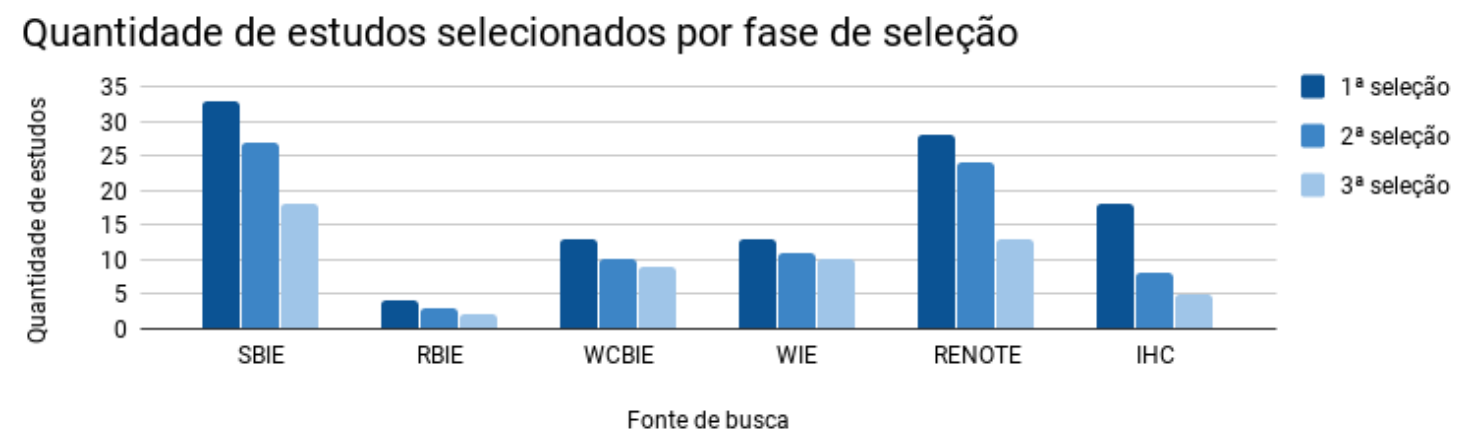

Figura 1. Resultados das seleções de estudos primários.

De posse dos estudos selecionados, com o intuito de gerar subsídios para responder as questões de pesquisa, cujos resultados são apresentados na próxima seção, foram realizadas a extração e posterior síntese dos dados, por meio de um formulário de coleta das informações mais relevantes acerca de cada estudo. Os artigos que passaram por esta triagem podem ser visualizados em uma planilha online ${ }^{1}$, onde estão separados por fonte de dados. Nos estudos excluídos, constam os critérios de exclusão.

\section{Resultados e Discussões}

Esta seção tem como objetivo buscar responder, individualmente, as questões de pesquisa discutindo seus resultados. As quatro questões definidas foram respondidas com base no levantamento realizado, constituindo, assim, os resultados desta RSL.

QP1: “Que tecnologias têm sido utilizadas e desenvolvidas para auxiliar na inclusão educacional de deficientes auditivos?"

A partir dessa revisão foi possível identificar diversificadas soluções tecnológicas com o propósito de auxiliar no processo de ensino e aprendizagem de deficientes auditivos no Brasil. Entre as soluções, 9 (nove) estudos relataram sobre a

\footnotetext{
${ }^{1}$ https://goo.gl/xvLfiZ
} 
VII Congresso Brasileiro de Informática na Educação (CBIE 2018)

Anais do XXIX Simpósio Brasileiro de Informática na Educação (SBIE 2018)

utilização de tecnologias já existentes, todos contendo algum tipo de resultado, como análises qualitativas dos softwares ou relatórios das percepções de usuários reais. Além disso, 43 estudos abordaram o desenvolvimento de novas soluções, dos quais 28 apresentaram resultados, como validação ou avaliação do software desenvolvido. Foram encontradas, também, 5 propostas de desenvolvimento de tecnologias, envolvendo projetos em andamento que, possivelmente, foram concretizados após serem publicados.

Assim, a partir da síntese dos dados acerca das categorias dessas tecnologias, foi possível elencar quais tecnologias têm sido mais utilizadas e desenvolvidas com o referido propósito. Esse resultado pode ser visualizado na Figura 2, a qual demonstra que as tecnologias que estão sendo mais utilizadas são os Tradutores LIBRAS-Língua Portuguesa e que as mais desenvolvidas são, em sua maioria, Jogos Educacionais, podendo-se destacar também as Arquiteturas Pedagógicas e os Ambientes Virtuais de Aprendizagem (AVA), sendo que esta última categoria corresponde também à maioria das propostas ainda não consolidadas no momento da publicação.

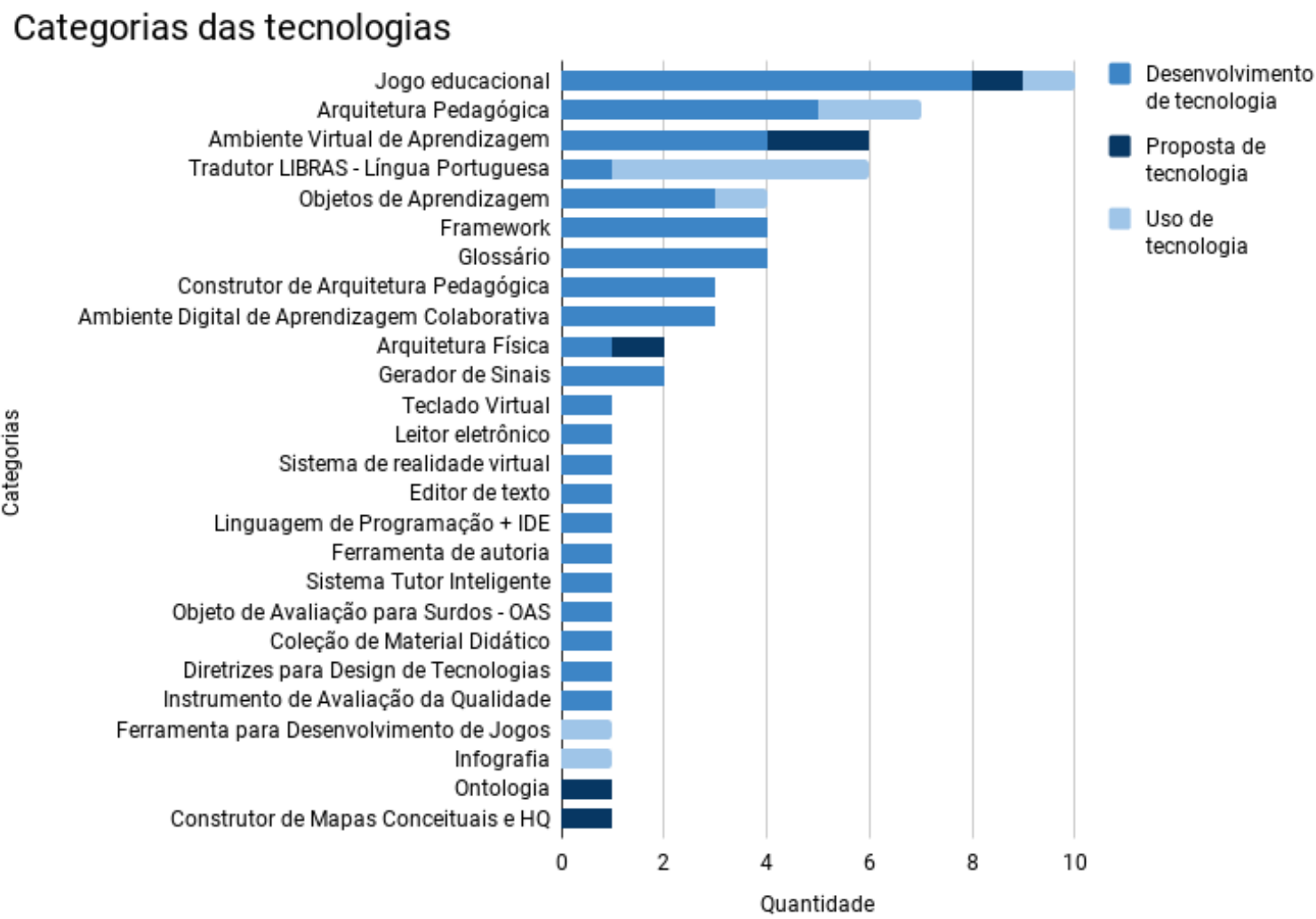

Figura 2. Categorias das tecnologias propostas, desenvolvidas ou utilizadas.

\section{QP2: “Quais os principais objetivos das tecnologias identificadas na QP1?"}

Os principais objetivos das tecnologias identificadas, elencados a partir da análise dos trabalhos que as propõem, concentram-se, como é possível visualizar na Figura 3, em auxiliar no ensino e aprendizagem de LIBRAS e Língua Portuguesa, sendo que a maioria propõe promover a educação bilíngue, com a oferta de ensino de LIBRAS e de Língua Portuguesa como segunda língua escrita.

Considerando o objetivo dessa RSL, destaca-se entre os trabalhos algumas tecnologias desenvolvidas para auxiliar no ensino e aprendizagem na área de 
VII Congresso Brasileiro de Informática na Educação (CBIE 2018)

Anais do XXIX Simpósio Brasileiro de Informática na Educação (SBIE 2018)

computação. Uma delas foi apresentada por [Santos et al. 2011], uma linguagem de programação baseada em LIBRAS (PROGLIB), criada a partir de um framework simplificado apoiado por uma IDE com suporte de um intérprete virtual. O experimento realizado com alunos do ensino fundamental e médio, possibilitou observar a facilidade dos alunos em utilizar a linguagem mesmo com pouca experiência. Outra contribuição que visa apoiar o ensino em Computação é apresentada por [Gonçalves et al. 2015] que buscaram promover capacitação profissional ao ofertar cursos EAD de programação em Java acessíveis para pessoas surdas. Para isso, foram produzidas e disponibilizadas videoaulas em um AVA. No momento da publicação, a oferta dos cursos estava em andamento e uma análise qualitativa seria conduzida futuramente.

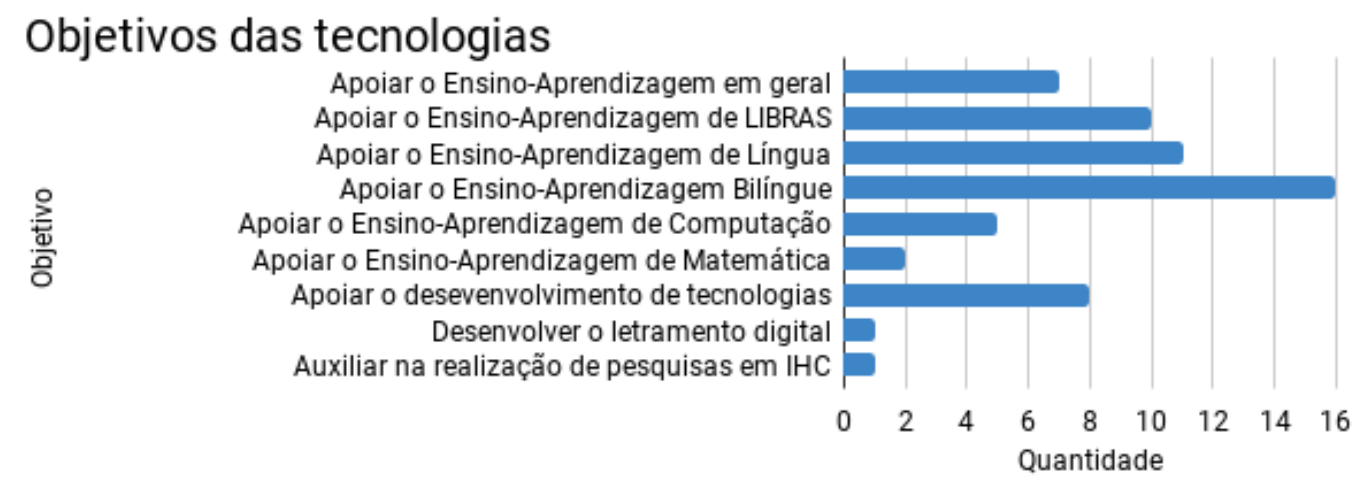

Figura 3. Objetivos das tecnologias propostas, desenvolvidas ou utilizadas.

Além dos anteriores, dois trabalhos mais recentes preocuparam-se em eliminar barreiras linguísticas, por meio de glossários com termos específicos de computação. Com o desenvolvimento do aplicativo LibrasTI ${ }^{2}$ [da Cruz et al. 2017] procuraram preencher as lacunas existentes de ferramentas de ensino com foco na área da Computação. Com objetivos semelhantes, [Granada et al. 2017] constituíram um glossário com as principais palavras reservadas da linguagem LOGO, de modo a estimular a lógica de computação e promover a interação tecnológica de alunos surdos, em parceria com uma escola bilíngue de ensino fundamental.

\section{na QP1?"}

QP3: “Que níveis de ensino são público-alvo das tecnologias identificadas

Como visto na questão anterior, a maioria das tecnologias visa auxiliar no Ensino-Aprendizagem de LIBRAS e Língua Portuguesa. Sabe-se que grande parte dos deficientes auditivos enfrenta dificuldades de alfabetização até a vida adulta, mas procurando atenuar essa situação, medidas têm sido tomadas no Ensino Fundamental, período em que as crianças são alfabetizadas. Isso se reflete nos trabalhos analisados que, em sua maioria, envolvem tecnologias desenvolvidas para este nível educacional. Além disso, há uma grande quantidade de estudos que não definem um nível de ensino em específico como público-alvo, visto que são propostos para qualquer usuário.

Percebeu-se poucas ocorrências de estudos direcionados ao Ensino Superior (apenas 2). Dentre estas, cita-se a plataforma web proposta por [Sarmento and do Rego 2017], uma arquitetura pedagógica direcionada à formação profissional de alunos com

\footnotetext{
${ }^{2}$ http://r1.ufrrj.br/petsi/librasti/
} 
VII Congresso Brasileiro de Informática na Educação (CBIE 2018)

Anais do XXIX Simpósio Brasileiro de Informática na Educação (SBIE 2018)

deficiência visual e auditiva em um curso de Operador de Microcomputador, que oferece material didático no formato de videoaula, em Libras e com áudio-descrição. [Lapolli et al. 2014] apresentam uma infografia na web que apresenta conceitos relativos a Geometria Descritiva (GD), e também relacionados à projeção cilíndrica ortogonal. Esta infografia foi avaliada por meio de entrevistas com um grupo de graduandos surdos, aonde averiguou-se que esta ferramenta pode ser utilizada para o ensino e aprendizagem, visto que os alunos a consideraram uma ferramenta pedagógica interessante devido ao forte apelo visual aliado a utilização de textos curtos.

\section{QP4: “Que contribuições são destacadas na educação de deficientes auditivos mediada por tais tecnologias?"}

Para elencar as principais contribuições que são destacadas ao utilizar tecnologias na educação de deficientes auditivos, foram investigados os estudos que apresentam as tecnologias que mais estão sendo desenvolvidas e utilizadas, segundo o levantamento apresentado na Figura 2.

Entre os jogos educacionais, [Rocha et al. 2017] propôs um jogo que utiliza signos para promover a aprendizagem, cujos resultados permitiram identificar benefícios na comunicação entre os alunos e professores, ressaltando-se a importância da intervenção do professor como mediador. [Carvalho and de Jesus Ferreira 2011] apresentaram um jogo que conduz atividades pedagógicas voltado ao desenvolvimento do vocabulário de alunos surdos e com a sua utilização verificou-se a promoção do reconhecimento da escrita da língua portuguesa pelos alunos. De forma geral, a partir da análise dos estudos que apresentaram jogos educacionais, evidenciou-se o potencial educador de ferramentas que tratam a aprendizagem de forma lúdica, destacando-se que estes podem ser utilizados por professores como meios de comunicação, expressão, aprendizagem e interação dentro da sala de aula.

Outra tecnologia que recebeu bastante atenção foi a arquitetura pedagógica, cujo principal papel verificado é a realização de tarefas escolares em ambientes computacionais, mediadas por professores em sala de aula com o objetivo de reforçar ainda mais as suas práticas pedagógicas. Além disso, estão sendo desenvolvidos construtores de arquiteturas pedagógicas, verificando-se o interesse em promover a elaboração de novas arquiteturas pedagógicas, viabilizadas também a usuários leigos em desenvolvimento de sistemas, como professores da rede básica de ensino.

Já entre os estudos que apresentam as tecnologias mais utilizadas, os tradutores LIBRAS-Língua Portuguesa, [Côrrea et al. 2014] analisaram conhecidos aplicativos de tradução, o ProDeaf $\mathrm{Móvel}^{3}$ e o HandTalk ${ }^{4}$, demonstrando a partir de uma pesquisa qualitativa que eles têm sido empregados como mediadores de interações linguísticas, possuindo caráter mediativo-inclusivo nas relações comunicativas. Outra tecnologia semelhante foi apresentada em [Moreira et al. 2011], o Player Rybená ${ }^{5}$, um tradutor cujo objetivo é mediar o processo de comunicação de forma mais próxima a realidade.

\footnotetext{
${ }^{3} \mathrm{http}: / /$ www.prodeaf.net/

${ }^{4} \mathrm{https}: / /$ www.handtalk.me/

${ }^{5} \mathrm{http}: / /$ portal.rybena.com.br/site-rybena/
} 
VII Congresso Brasileiro de Informática na Educação (CBIE 2018)

Anais do XXIX Simpósio Brasileiro de Informática na Educação (SBIE 2018)

\section{Considerações finais}

A partir da revisão aqui apresentada, verificou-se que é crescente o interesse em desenvolver novas tecnologias que possam auxiliar na inclusão e promoção da aprendizagem de deficientes auditivos. Infelizmente, de acordo com o levantamento, as tecnologias mais utilizadas no Brasil com esse objetivo não foram desenvolvidas para serem empregadas especificamente em sala de aula.

Espera-se, então, que as tecnologias que estão sendo constantemente desenvolvidas sejam disponibilizadas para utilização em âmbito escolar, pois de acordo com os resultados dos estudos examinados, verifica-se que os objetivos das ferramentas computacionais propostas são mais eficientes quando aplicadas em sala de aula. Ademais, utilizando-as nesse contexto verifica-se a eficácia do caráter inclusivo, devido a mediação do professor e a importante participação dos colegas nesse processo.

Importantes soluções tecnológicas foram identificadas com a análise de cada trabalho, a partir das quais foram elencadas contribuições e limitações, já relatadas aqui. Para complementar esse conhecimento, deseja-se, com o auxílio de intérpretes, entrevistar alunos de graduação com deficiência auditiva, para descobrir as principais barreiras de aprendizagem e inclusão que são enfrentadas no seu dia-a-dia. Finalmente, de posse dessas informações, pretende-se propor uma ou mais intervenções tecnológicas para promover a inclusão educacional dos deficientes auditivos no ensino superior.

\section{Agradecimentos}

Nossos agradecimentos ao Programa de Educação Tutorial - PET/MEC, pelo apoio na realização desse trabalho.

\section{Referências}

Brasil (1988). "Constituição da República Federativa do Brasil de 1988". http://www.planalto.gov.br/ccivil 03/constituicao/constituicao.htm. Jun. 2018.

Brasil (2008). "Política Nacional de Educação Especial na Perspectiva da Educação Inclusiva”. MEC/SEESP.

Brasil (2012). "Lei n. 12.711, de 29 de agosto de 2012". http://www.planalto.gov.br/ccivil 03/ ato2011-2014/2012/lei/112711.htm. Jun. 2018.

Carvalho, N. A. and de Jesus Ferreira, B. (2011). "Projeto e implementação de uma ferramenta voltada ao desenvolvimento do vocabulário em língua portuguesa de crianças surdas". RENOTE, 9(3).

Corrêa, Y., Vieira, M. C., Santarosa, L. M. C., and Biasuz, M. C. V. (2014). "Aplicativos de tradução para libras e a busca pela validade social da tecnologia assistiva". Anais do XXV SBIE.

da Cruz, S. M. S., Calé, Rodrigues, F., Miranda, R. C. T., Volpasso, L. H. S., Paim, L. N., and Benassi, P. M. (2017). "Uma ferramenta para auxiliar o ensino da tecnologia da informação para surdos". Anais dos Workshops do VI CBIE (WCBIE).

da Silva, R. Q., Guarinello, A. C., and de Oliveira Martins, S. E. S. (2016). "O intérprete de libras no contexto do ensino superior". Revista Teias, 17(46). 
VII Congresso Brasileiro de Informática na Educação (CBIE 2018)

Anais do XXIX Simpósio Brasileiro de Informática na Educação (SBIE 2018)

de Souza, J. H. S., Guilherme, I. R., Borges, S. d. S., Filho, R. R. R., and Reis, H. M. (2016). "Softwares de geometria interativa para deficientes físicos e intelectuais: Um mapeamento sistemático". RENOTE, 14(2).

Gonçalves, E. J. T., Oliveira, F., Vilela, J. F., Peixoto, M. M., and Castro, J. B. (2015). "Produção de videoaulas de programação em java acessíveis no contexto de um projeto de capacitação profissional para pessoas surdas". Anais do XXVI SBIE.

Granada, R. P., Cesário, V. M., Domingues, D., Barwaldt, R., Nagel, R., and Fernandes, C. L. (2017). "Dicionário de termos de computação como facilitador no ensino de programação para surdos'. Anais dos Workshops do VI CBIE (WCBIE).

INEP (2018). "Resumo Técnico Censo Educação Superior 2015”. http://inep.gov.br/resumos-tecnicos1. Jun. 2018.

Kitchenham, B. (2004). "Procedures for performing systematic reviews". Technical Report. Department of Computer Science, Keele University, UK.

Lapolli, M., Vanzin, T., and Ulbricht, V. R. (2014). "Infografia na web para a inclusão digital de alunos surdos". RENOTE, 12(1).

Moreira, J. R., Ferneda1, E., Brito, P. H., Coradine, L. C., Guadagnin, R. d. V., de Oliveira, R. M., and de Vilhena Garcia, E. (2011). "Rumo a um sistema de tradução português-libras". Anais do XXII WIE.

ONU (1948). "Declaração Universal dos Direitos Humanos". https://www.ohchr.org/ EN/UDHR/Documents/UDHR Translations/por.pdf. May. 2018.

ONU (1994). "Declaração de Salamanca Sobre Princípios, Políticas e Práticas na Área das Necessidades Educativas Especiais". http://portal.mec.gov.br/seesp/ arquivos/pdf/salamanca.pdf. Jun. 2018.

Ramires, L. O., Souza, E. P., de Souza, J. N., R., F. M., Janurio, M. V., Silva, R. E., Gomes, R. S., and de Lima, T. A. (2013). "Como a tecnologia assistiva tem auxiliado o processo de ensino/aprendizagem? mapeamento sistemático a partir dos trabalhos publicados no sbie". Anais do XXIV SBIE.

Rocha, D., Bittencourt, I. I., Amorim, R., and Ospina, P. (2017). "Uma tecnologia assistiva baseada na semiótica peirceana para a educação inclusiva de crianças surdas e ouvintes". Anais dos Workshops do VI CBIE (WCBIE).

Rocha, D. F. S., Bittencourt, I. I., Dermeval, D., and Isotani, S. (2014). "Uma revisão sistemática sobre a educação do surdo em ambientes virtuais educacionais". Anais do XXV SBIE.

Santos, F. T., Coutinho, F. J., and Brito, P. H. d. S. (2016). "Uma revisão sistemática sobre softwares educacionais para o ensino de libras". Anais do XXVII SBIE.

Santos, R. E. S., Magalhães, C. V. C., Neto, J. S. C., and Jú, S. S. L. P. (2011). "Proglib: Uma linguagem de programação baseada na escrita de libras". Anais do XXII WIE.

Sarmento, C. F. and do Rego, H. C. (2017). "Acessibilidade e inclusão digital de cegos e surdos em plataformas web: um estudo de casos". Anais do XXVIII SBIE.

Unesco (1998). "Declaração Mundial sobre Educação para Todos: Satisfação das necessidades básicas de aprendizagem Jomtien, 1990". 